\title{
Pure imagery hemi-neglect of far space
}

\author{
S. Ortigue, MS; I. Viaud-Delmon, PhD; C.M. Michel, PhD; O. Blanke, MD; J.M. Annoni, MD; \\ A. Pegna, PhD; E. Mayer, PhD; L. Spinelli, PhD; and T. Landis, MD
}

\begin{abstract}
Patients with hemispatial neglect restricted to near (within reaching distance) or to far space (beyond reaching distance) have been described. This constitutes a double-dissociation considered by current neurocognitive thinking as compelling evidence for separate networks. However, a similar double-dissociation exists with respect to perceived as opposed to imagined space. If the organization of represented space was similar to that of perceived space, it should contain a far/near dissociation as well. This paper describes a patient with pure representational neglect restricted to far space.
\end{abstract}

NEUROLOGY 2003;60:2000-2002

Differences in patients' handling of space within $^{1}$ or beyond ${ }^{2}$ reaching distance have puzzled clinicians for more than half a century. ${ }^{1-5}$ Recent descriptions of patients with hemispatial neglect restricted to near ${ }^{1}$ or far perceptual space ${ }^{2}$ supported the assumption of specific neural networks dedicated to treating different portions of space. Studies in monkeys provide neurophysiological support for this dissociation. Ablation of area 8 led to an attentional deficit within contralesional space beyond reaching distance (far space), whereas ablation of area 6 led to a specific deficit within reaching distance (near space). ${ }^{5}$

Equally puzzling is the dissociation observed between some neglect patients' treatment of physically perceived vs mentally imagined (representational) space. The behavior of two patients reported in the 1970s provided the first description of representational neglect. ${ }^{6}$ They were asked to imagine ("seeing" in the absence of the corresponding sensory input) a familiar city square from two opposite viewercentered reference frames. Both patients could describe only the right half of the square with respect to their imagined viewpoint. Thus, despite knowing the whole city square, they systematically omitted the left half in a viewer-centered reference frame.

Rapidly different patients were discovered who had a pure perceptual neglect, ${ }^{3}$ but also some rare patients with a pure representational neglect. ${ }^{7}$ There is an old question whether perception is organized similarly to mental representation. ${ }^{8}$ If space is indeed fragmented in different compartments according to the reaching-distance in perceptual domain, we would expect to find the same near vs far distinction in purely representational space. ${ }^{9}$ We tested this hypothesis in a patient with pure representational neglect.
Patient and methods. This 78-year-old right-handed woman had a hemorrhage of the right lateral temporo-occipital junction (figure 1). Neurologic examination findings were normal, except for a left-sided hyperreflexia. She was oriented and had no visual field deficit. Detailed neuropsychological examination findings were normal, except for a mild impaired figural fluency, discrete difficulties with nonconventional views of objects, and minimal difficulties in learning visuospatial (Rey signs) and verbal (Rey words) information with intact recognition memory. Her shortterm memory was in the normal range for verbal (digit span) and visuospatial modalities (Corsi block-tapping span). There was no language impairment (Boston Naming Test, repetition, reading, writing, and comprehension), apraxia, visual agnosia, or dysexecutive disorder.

We tested perceptual and representational spaces in the following four, counterbalanced conditions.

Condition 1. We assessed near perceptual space with four standard tasks using paper and pencil (line bisection and three cancellation tasks using letters, digits, and lines). We also tested her in a landscape-copying task, a star cancellation task, a clockcopying task, sentence reading, and the "fluff test" (with eyes closed).

Condition 2. We assessed far perceptual space with the same four standard line bisection and cancellation tasks, as described for near perceptual space, except that stimuli appeared at $\sim 3.5 \mathrm{~m}$ distance from the patient, who now used a laser pointer to respond.

Condition 3. We assessed near representational space with two tests investigating the space within her imagined reaching distance. In the first test, the patient was asked to imagine herself inside her car and to describe what she would see from two successively given viewpoints: from the passenger's seat (i.e., the majority of the dashboard on her left), and from the driver's seat (i.e., the majority of the dashboard on her right). Her description was recorded for 1 minute and was rated with reference to six main items on each side of the given viewpoint, which are commonly present in a car (figure $2 \mathrm{~A}$ and $2 \mathrm{~B}$ ). In the second test (object layout), she was instructed to carefully look at a number of objects (three per side) placed within reach on the table in front of her. She was then blindfolded and asked to recall, immediately and after 15 minutes' delay, as many objects' locations as possible from two opposite imposed viewpoints.

Condition 4. We assessed far representational space with two standard tests considered to probe beyond the reaching distance of the hypothetical viewpoint of the patient. In the first (familiar square description), the patient was asked to imagine the "Place Neuve" (a familiar square in Geneva ${ }^{7}$ ) and to describe what she would see from two opposite imposed viewpoints. In the second

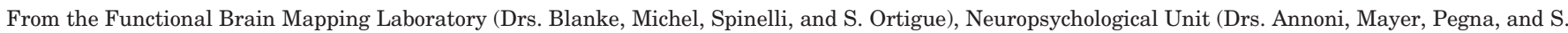

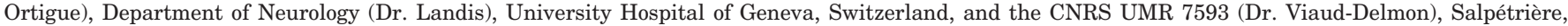
Paris, France.

Supported by The Swiss National Research Foundation grant \#3100-065096.01.

Received October 17, 2002. Accepted in final form February 21, 2003.

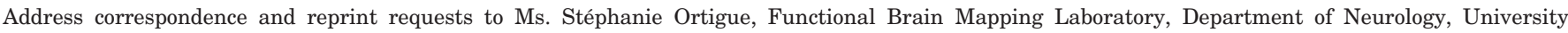
Hospital of Geneva, 24 rue Micheli-du-Crest, CH-1211 Geneva 14, Switzerland; e-mail: stephanie.ortigue@hcuge.ch 

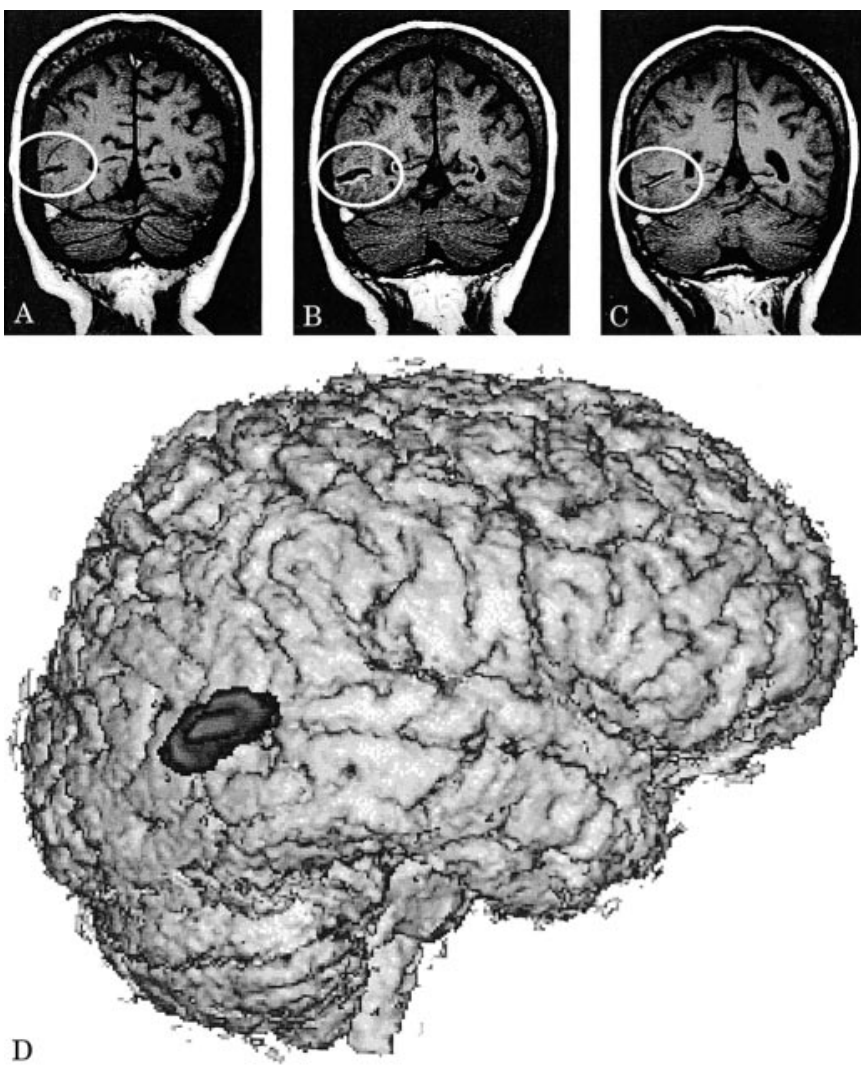

Figure 1. (A-C) Coronal slices of T1-weighted MR images $(0.98 \mathrm{~mm}$ thick) and $(D)$ three-dimensional brain reconstruction, showing the patient's hemorrhagic lesion involving the right lateral temporo-occipital junction.

test (familiar map description), the patient was asked to imagine the map of France as if she were a giant able to see very far and name as many towns as possible from two opposite imposed viewpoints (Paris and Marseille). This was done in order to reduce the probability that she would behave as if she were holding a map in her hands and rotating it.

Results. This two-by-two design modified either the nature of the investigated space (perceptual or representational) or the reaching distance (within or beyond). The patient showed a left representational neglect only for far space in the absence of any perceptual neglect. Indeed, she perceptually attended to all objects presented, either to her right or left hemispace, both within (condition 1) or beyond (condition 2) her reach. There was no significant deviation in line bisection (near: mean deviation $=-1 \mathrm{~mm} \pm$ 3; far: mean deviation $=-3 \mathrm{~mm} \pm 1$ ) and no omission in the cancellation tasks. Copying, reading tasks, and the "fluff test" (with eyes closed) were not impaired by neglect-related errors.

Assessment of her near (within reaching distance) imagined space (condition 3) did not reveal any neglect omission (table). In contrast, when she mentally imagined a familiar scene/map (condition 4), she only reported elements located to the right of her hypothetical viewpoint, systematically omitting those on her left side. In the familiar square description, she reported six buildings on her right vs none on her left from both imagined viewpoints (see figure $2 \mathrm{C}$ ). In the familiar map description, she named six cities on her left according to a hypothetical axis between Marseille and Paris, against 12 on her right (McNemar $\chi^{2}=11.47$, $\mathrm{p}=0.0007$ ). From the $180^{\circ}$-rotated imagined viewpoint, she listed four cities to the left of her imaginary viewpoint vs 10 to the right (McNemar $\left.\chi^{2}=15.56, p=0.0001\right)$. This mental deficit was thus restricted to the imagined descriptions supposed to be far away from her reaching distance.
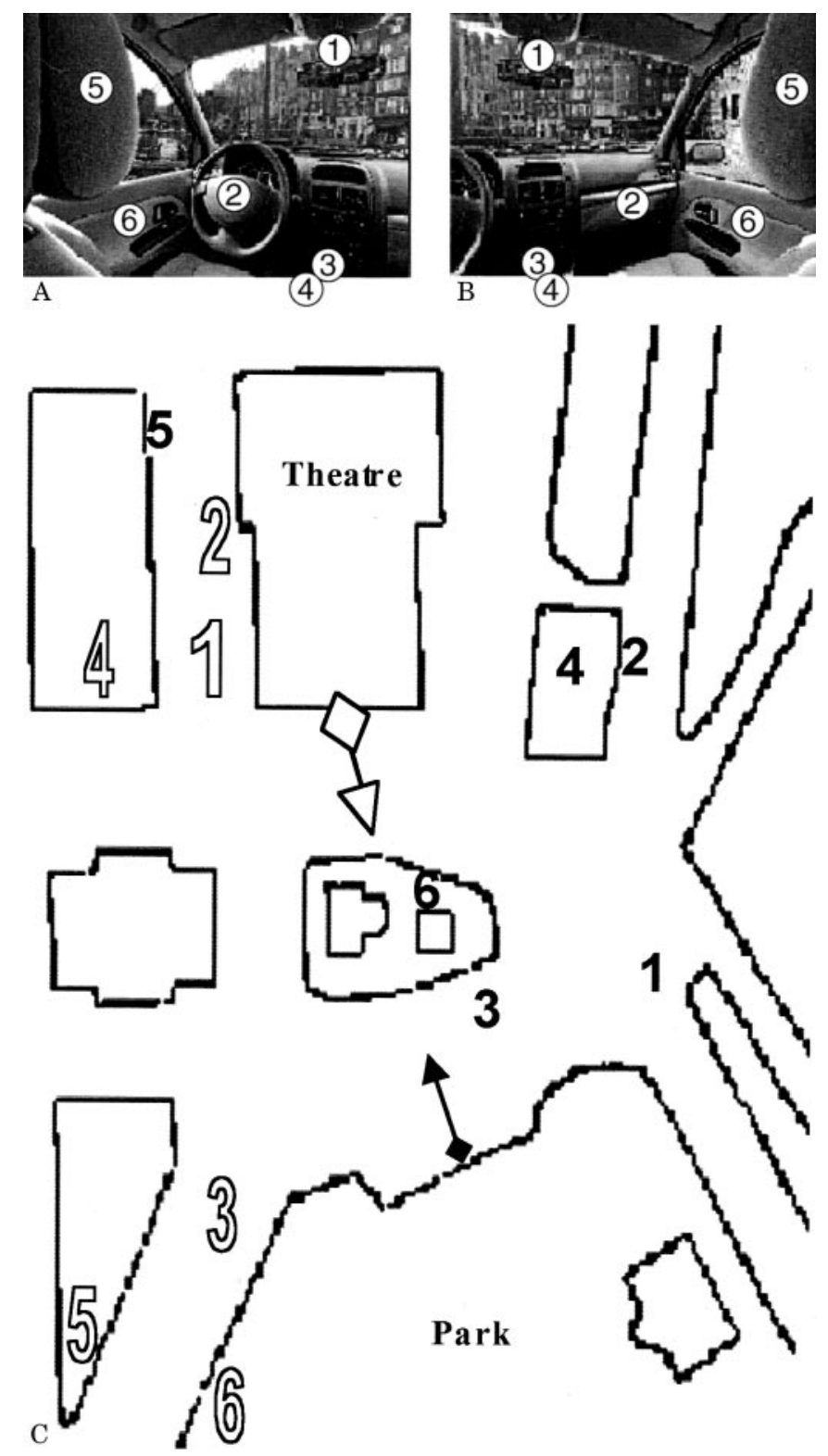

Figure 2. Inside car test. These photographs indicate what the patient might have imagined seeing (numbers indicate locations of six main objects used for reference in rating her performance) from (A) the passenger's side $(1=$ rear-view mirror, 2 = steering wheel, 3 = gearbox, $4=$ handbrake, 5 = seat, 6 = left door) or (B) driver's side ( 1 = rear-view mirror; 2 = glove compartment, $3=$ gearbox, 4 = handbrake, 5 = seat, 6 = right door). (C) Maps of a familiar square in Geneva as described from mental imagery. The position of each viewpoint is indicated by a square with an arrow pointing in the imagined viewing direction. Digits indicate locales reported by the patient and are ordered according to the sequence in which they were reported. Their shading corresponds to the imposed viewpoint imagined by the patient at the time of test.

Discussion. These results provide clinical information about the cerebral organization of imagined (representational) space. This is of particular importance, because the mental representation of space is difficult to assess in nonhuman primates. 
Table Performance on tasks testing near and far representational space for each of the two opposite imposed viewpoints

\begin{tabular}{|c|c|c|c|c|c|c|c|c|}
\hline & \multicolumn{4}{|c|}{ Far space } & \multicolumn{4}{|c|}{ Near space } \\
\hline & \multicolumn{2}{|c|}{ The "Place Neuve" } & \multicolumn{2}{|c|}{ The map of France } & \multicolumn{2}{|c|}{ Object layout } & \multicolumn{2}{|c|}{ Inside of car } \\
\hline $\begin{array}{l}\text { First } \\
\text { viewpoint }\end{array}$ & $0 \%(0 / 6)$ & $100 \%(6 / 6)$ & $38 \%(6 / 16)$ & $75 \%(12 / 16)$ & $100 \%(3 / 3)$ & $100 \%(3 / 3)$ & $100 \%(6 / 6)$ & - \\
\hline
\end{tabular}

The percentage (referenced to items reported in the nonneglected side) and number correct are shown.

Anatomically, both clinical studies as well as experiments in healthy subjects suggest that the perception of far space depends on temporal areas, ${ }^{2,10}$ whereas near perceptual space depends more on parietal areas. ${ }^{1,10}$ Brain damage in the few patients with pure representational neglect does not suggest one single location and was found after frontal, frontoparietal, or thalamic lesion in the right hemisphere. ${ }^{7}$ Our patient showed severe viewer-centered spatial neglect selective for far representational space after a right lateral posterior temporal lesion. Access to the mental representation of near "grasping" space thus remained intact. This can either be due to a selective impairment of networks coding for the representation of far space, or can alternatively be due to the fact that far space is only visually coded, whereas the representation of near space has a visual and a motor memory component. Our data suggest the following. First, whereas mental imagery and sensory perception have distinct ways of accessing stored visual representations, they share some conceptual mechanisms, at least along a far vs near dimension. Second, temporal areas might be important for the mental representation of far space when a viewer-centered reference is imposed. Finally, our findings call for an eventual discovery of a doubledissociation, i.e., a patient with pure representa- tional neglect only for near space, after rather a right parietal than a temporal lesion.

\section{Acknowledgments}

The authors thank Micah M. Murray for his helpful comments on the manuscript.

\section{References}

1. Halligan PW, Marshall JC. Left neglect for near but not far space in man. Nature 1991;350:498-500.

2. Vuilleumier P, Valenza N, Mayer E, Reverdin A, Landis T. Near and far visual space in unilateral neglect. Ann Neurol 1998;43:406-410.

3. Grüsser OJ, Landis T. Visual agnosias and other disturbances of visual perception and cognition, Chapter 22: Only half of the word: Visual hemineglect and related disorders. In: Cronly-Dillon JR, ed. Vision and Visual Dysfunction. Vol. 12. London: MacMillan, 1991;431-466.

4. Brain WR. Visual disorientation with special reference to lesions of the right cerebral hemisphere. Brain 1941;64:244-272.

5. Rizzolatti G, Matelli M, Pavesi G. Deficits in attention and movement following the removal of postarcuate (area 6) and prearcuate (area 8) cortex in macaque monkeys. Brain 1983;106:655-673.

6. Bisiach E, Luzzatti C. Unilateral neglect of representational space. Cortex 1978;14:129-133.

7. Ortigue S, Viaud-Delmon I, Annoni JM, et al. Pure representational neglect after right thalamic lesion. Ann Neurol 2001;50:401-404.

8. Bartolomeo P. The relationship between visual perception and visual mental imagery: a reappraisal of the neuropsychological evidence. Cortex 2002;38:357-378.

9. Landis T. Disruption of space perception due to cortical lesions. Spat Vis 2000;13:179-191.

10. Previc FH. Functional specialization in the lower and upper visual fields in humans: its ecological origins and neurophysiological implications. Behav Brain Sci 1990;13:519-575. 